\title{
Frequiência da Infecção pelo Papilomavírus Humano em Mulheres com Ectopia Cervical
}

\author{
Frequency of Human Papillomavirus Infection in Women with Cervical Ectopia
}

Eddie Fernando Candido Murta, Welington Lombardi, Luciana Souza Borges

Maria Azniv Hazarabedian de Souza, Sheila Jorge Adad

\begin{abstract}
RESUMO
Objetivo: fatores como múltiplos parceiros sexuais, idade, inicio precoce da atividade sexual, fumo e uso de anticoncepcional oral (ACO) têm sido relacionados com a maior incidência de infecção pelo papilomavírus humano (HPV). A presença de ectopia cervical também tem sido relacionada, embora com resultados contraditórios. $O$ objetivo deste é analisar um grupo de mulheres com infecção pelo HPV e verificar a incidência de ectopia cervical.

Métodos: foram estudadas 471 mulheres com diagnóstico de infecção pelo HPV por meio da citologia (critério de Schneider et al.) e a relação com a ectopia cervical, uso de ACO e a sexarca.

Resultado: dos 471 casos estudados, 182 (38,6\%) apresentavam ectopia. Das 182 pacientes com ectopia, 157 (86,3\%) tinham idade igual ou inferior a 30 anos, ao passo que 47,8\% das pacientes sem ectopia pertenciam a esse grupo etário $(p<0,001)$. Quanto ao início da atividade sexual, não houve diferença significante entre os grupos: das pacientes com ectopia, 77,4\% tinham 18 anos ou menos, em comparação com 71,3\% no grupo sem ectopia. Dos casos com ectopia, 45,7\% estavam em uso de ACO, enquanto apenas $24,3 \%$ das pacientes sem ectopia utilizavam esse método $(p<0,001)$.

Conclusões: conclui-se que a ectopia não está associada à sexarca e a prevalência de ectopia nas pacientes com HPV foi maior no grupo com menos de 30 anos e/ ou em uso de ACO.
\end{abstract}

PALAVRAS-CHAVE: HPV. Colo do útero: lesões pre-neoplásicas. Colposcopia. Colpocitologia. Contracepção.

\section{Introdução}

O papilomavirus humano (HPV) é um DNA virus epiteliotrófico, que está sendo vinculado à carcinogênese do colo uterino por meio de evidências epidemiológicas e laboratoriais ${ }^{1}$. Deste vínculo emerge o grande interesse em se estabelecerem fatores de risco para a infecção que causa, a fim de se poder detectar as populações

Disciplina de Ginecologia e Obstetrícia da Faculdade de Medicina do Triângulo Mineiro.

Correspondência:

Eddie Fernando Candido Murta

Disciplina de Ginecologia e Obstetrícia da Faculdade de Medicina do Triângulo Mineiro.

Av. Getúlio Guaritá, S/N

38025-440 - Uberaba - MG

e-mail: eddiemurta@mednet.com.br sujeitas a carcinogênese cervical induzida pelo HPV.

A infecção pelo HPV é diagnosticada mais freqüentemente durante a gravidez, em mulheres jovens com múltiplos parceiros sexuais, com início da atividade sexual antes dos 18 anos de idade, nas fumantes e usuárias de anticoncepcionais hormonais ${ }^{2,3,4,5,6,7}$. A ectopia cervical é outro fator que tem sido relacionado com a infecção por HPV. Toon et $a .^{8}$ demonstraram que mulheres com citologia normal e ectopia apresentam mais freqüentemente HPV-DNA que as pacientes sem ectopia. Não obstante, em um estudo realizado por Karlsson et al. ${ }^{3}$, não se encontrou relação entre infecção por HPV e ectopia.

O objetivo deste trabalho foi avaliar a freqüência de ectopia em mulheres com sinais 
citológicos de infecção por HPV e a relação entre a ectopia cervical e o uso de anticoncepcional oral (ACO) e o início da atividade sexual.

\section{Pacientes e Métodos}

Foram revistos 471 laudos colposcópicos de pacientes com diagnóstico citológico de infecção por HPV, apresentando ou não neoplasia intraepitelial cervical grau I (NIC I). Estas pacientes foram atendidas no Ambulatório de Oncologia Ginecológica da Disciplina de Ginecologia e Obstetrícia da Faculdade de Medicina do Triângulo Mineiro no período de julho de 1.993 a dezembro de 1.997. O diagnóstico citológico foi feito conforme padronizado em nosso Serviço e segue os critérios de Schneider et al. ${ }^{9}$, que se baseiam na presença de coilocitose clássica ou na presença de seis ou mais dos nove critérios não-clássicos, que são: coilocitose leve, disqueratocitose leve, citoplasma claro, grânulos cerato-hialinos, estriação citoplasmática, células fusiformes disceratóticas, hipercromasia nuclear, bi ou multinucleação e halo perinuclear. Nenhuma das pacientes tinha sido submetida a qualquer tratamento. As pacientes com diagnóstico de NIC II ou III foram excluídas da análise. Os dados coletados foram a idade da paciente, a idade de início da atividade sexual e o uso de ACO. Na análise estatística utilizou-se o teste do $\chi^{2}$ e foi considerado significante para $\mathrm{p}<0,05$.

\section{Resultados}

Dos 471 casos com diagnóstico citológico de infecção pelo HPV associado ou não ao de NIC I, $182(38,6 \%)$ apresentavam ectopia com a junção escamocolunar (JEC) estendendo-se para fora do orificio externo. As demais 289 mulheres apresentavam a JEC coincidindo com o orificio externo do canal cervical (posição "0,0") ou no seu interior. A média de idade das 471 mulheres foi de $29,4 \pm 11,6$ anos. Entre as 182 pacientes com ectopia (idade média de $24 \pm 6,9$ anos), 157 (86,3\%) tinham idade igual ou inferior a 30 anos por ocasião do diagnóstico, ao passo que apenas $47,8 \%$ das pacientes sem ectopia (média de idade: $32,8 \pm 12,6$ anos) pertenciam a esse mesmo grupo etário ( $p<0,001$, Tabela 1). Em $77,4 \%$ dos casos com ectopia e em $71,3 \%$ dos casos que não apresentavam ectopia, a sexarca ocorreu com 18 anos ou menos (média de 17,4 $\pm 3,9$ anos), não sendo essa diferença estatisticamente significante (Tabela 2). Das pacientes com ectopia, 45,7\% estavam em uso de ACO, ao passo que $24,3 \%$ das pacientes sem ectopia, faziam uso desse método $(\mathrm{p}<0,001)$ (Tabela 3). A média de idade das pacientes que usavam ACO foi de 22,8 $\pm 5,7$ anos e a das que não faziam uso do método, foi de $27,9 \pm$ 6,8 anos.

Tabela 1 - Distribuição das pacientes segundo o grupo etário e a presença de ectopia.

\begin{tabular}{lcccc}
\hline & \multicolumn{2}{c}{ Com Ectopia } & \multicolumn{2}{c}{ Sem Ectopia } \\
Grupo Etário (anos) & $\mathbf{n}$ & $\%$ & $\mathbf{n}$ & $\%$ \\
\hline$\leq 30$ & 157 & 86,3 & 138 & 47,8 \\
$>30$ & 25 & 13,7 & 151 & 52,3 \\
Total & 182 & 100,0 & 289 & 100,0 \\
\hline$\chi^{2}=70,773$. Correção de Yates $=69,137, p<0,001$ & &
\end{tabular}

Tabela 2 - Distribuição das pacientes segundo o início da atividade sexual e a presença de ectopia.

\begin{tabular}{lcccc}
\hline & \multicolumn{2}{c}{ Com Ectopia } & \multicolumn{2}{c}{ Sem Ectopia } \\
Idade do primeiro coito (anos) & $\mathbf{n}$ & $\%$ & $\mathbf{n}$ & $\%$ \\
\hline$\leq 18$ & 13 & 77,4 & 194 & 71,3 \\
$>18$ & 39 & 22,6 & 78 & 28,7 \\
Total & 173 & 100,0 & 272 & 100,0
\end{tabular}

$\chi^{2}=2,052$. Correção de Yates $=1,748 . p=$ não-significante

Tabela 3 - Distribuição das pacientes segundo uso de anticoncepcional oral (ACO) e a presença de ectopia cervical.

\begin{tabular}{lcccc}
\hline & \multicolumn{2}{c}{ Com Ectopia } & \multicolumn{2}{c}{ Sem Ectopia } \\
ACO & $\mathbf{n}$ & $\%$ & \multicolumn{1}{c}{$\mathbf{n}$} & $\%$ \\
\hline Sim & 79 & 45,7 & 65 & 24,3 \\
Não & 94 & 54,3 & 203 & 75,7 \\
Total & 173 & 100,0 & 268 & 100,0 \\
\hline
\end{tabular}

$\chi^{2}=21,917$. Correção de Yates $=20,954 . p<0,001$

\section{Discussão}

Os resultados deste trabalho mostram que a ectopia cervical nas mulheres com sinais citológicos de infecção por HPV é mais comum abaixo dos 30 anos, o que está de acordo com os dados da literatura ${ }^{4,10}$.

Com relação ao fato de a ectopia papilar ser mais freqüente em mulheres que usam ACO, nossos dados confirmam os da literatura ${ }^{11}: 45,7 \%$ das mulheres com ectopia usavam ACO e apenas $24,3 \%$ das que não apresentavam ectopia faziam uso de tal medicação. Esta relação deve-se ao efeito estrogênico ${ }^{12}$.

Discute-se se as mulheres com ectopia 
cervical tem maior prevalência de infecção por HPV. Toon et al. $^{8}$ encontraram positividade para HPV DNA na cérvice de 14 de 49 pacientes com ectopia colunar (29\%), sendo que metade das 14 mulheres apresentava citologia normal. Os autores argumentam que possivelmente o HPV está hospedado nas erosões cervicais perto da junção escamocolunar após a transmissão pela atividade sexual em mulheres de risco ${ }^{13}$. Este sítio está ativamente envolvido na evolução da zona de transformação, submetida a um processo de metaplasia escamosa e que pode facilitar a entrada do virus no interior das células e, possivelmente em associação com outros agentes, iniciar a evolução para neoplasia intraepitelial cervical ${ }^{4}$.

Não obstante, Karlsson et al. ${ }^{3}$ estudando a positividade para HPV - DNA, verificaram que $20 \%$ das mulheres com ectopia apresentavam infecção por HPV comparadas com $24 \%$ que apresentavam esta infecção mas não tinham ectopia cervical, não sendo esta diferença estatisticamente significante. Estes autores concluíram que a ectopia não é fator predisponente para infecção por HPV.

Nossos resultados mostraram que $38,6 \%$ das pacientes com infecção pelo HPV tinham ectopia papilar. A comparação com os resultados da literatura mostra que a incidência relatada na literatura é menor em comparação ao nosso achado $^{4,5}$. Esta diferença pode ser explicada pela inclusão nos diversos trabalhos de grupos heterogêneos e pelos critérios diagnósticos para infecção pelo HPV.

Concluimos que a maioria das pacientes com sinais citológicos de infecção por HPV teve o primeiro coito antes dos 18 anos de idade, não sendo relacionado a presença ou não de ectopia. A prevalência de ectopia foi maior nas pacientes com menos de 30 anos e entre as que usavam ACO.

\section{SUMMARY}

Purpose: the number of male sexual partners, age, precocious beginning of sexual activity, cigarette smoking and oral contraception were correlated with human papillomavirus (HPV) infection, as were cervical ectopia, although with conflicting results. The objective is to analyze a group of women with HPV infection and to verify the incidence of cervical ectopia.

Methods: we have studied 471 women with Papanicolaou smears suggesting HPV infection (Schneider et al.'s criteria) and its relationship with cervical ectopia, beginning of sexual activity and the use of oral contraceptive.

Results: of the total of cases, 182 (38.6\%) had ectopia. Of these, $157(86.3 \%)$ were 30 years old or less, compared to $47.8 \%$ of women without ectopia $\left(p<0.001, \chi^{2}\right.$ test). A percentage of 77.4 of cases with ectopia had the beginning of sexual activity before 18 years compared to $71.3 \%$ cases without ectopia. Among women with ectopia, $45.7 \%$ had taken the pill recently compared to $24.3 \%$ which had not $(p<0.001$, $\chi^{2}$ test).

Conclusions: it was concluded that the beginning of sexual activity was not correlated with ectopia. The prevalence of ectopia was more commom in women under 30 years and/or in use of oral contraceptive.

KEY WORDS: HPV infection. Uterine cervix: preneoplastic lesions. Oral contraceptive.

\section{Referências}

1. Bosch FX, Munoz N, de Sanjose S, Izarzugaza I, Gili M, Viladiu $\mathrm{P}$, et al. Risk factors for cervical cancer in Colombia and Spain. Int J Cancer 1992; 52:750-8.

2. Gopalkrishna V, Murthy NS, Sharma JK, Roy M, Das DK, Luthra UK, et al. Increased human papillomavirus infection with the increasing number of pregnancies in Indian women. $J$ Infect Dis 1995;171:254-5.

3. Karlsson R, Jonsson M, Edlund K, Evander M, Gustavsson A, Bodén E, et al. Lifetime number of partners as the only independent risk factor for human papillomavirus infection: a population-based study. Sex Transm Dis 1995; 22: 119-27.

4. Koutsky LA, Galloway DA, Holmes KK. Epidemiology of genital human papillomavirus infection. Epidemiol Rev 1988; 10: 122-63.

5. Meisels A. Cytologic diagnosis of human papillomavirus: influence of age and pregnancy stage. Acta Cytol 1992;36:480-2.

6. Murta EFC, Souza MAH, Lombardi W, Borges LS Aspectos epidemiológicos da infecção pelo papilomavirus humano. J Bras Ginecol 1997; 107:95-9.

7. Schneider A, Hotz M, Gissmann L. Increased prevalence of human papillomaviruses in the lower genital tract of pregnant women. Int J Cancer 1987;40:198-201.

8. Toon PG, Arrand JR, Wilson LP, Sharp DS. Human papillomavirus infection of the uterine cervix of women without cytological signs of neoplasia. Br Med J 1986; 293: 1261-4.

9. Schneider A, Meinhardt G, De-Villiers EM, Gissmann L. Sensitivity of the cytologic diagnosis of cervical condyloma in comparison with HPV-DNA hybridization studies. Diagn Cytopathol 1987; 3: 250-5.

10. Singer A. The uterine cervix from adolescence to the menopause. Br J Obstet Gynaecol 1975; 82: 81-99.

11. de Villers EM, Schneider A, Miklaw H, Papendick U, Wagner D, Wesch $\mathrm{H}$, et al. Human papillomavirus infections in women with and without abnormal cervical cytoloy. Lancet 1987; 2: 703-6.

12. Gissmann L, Diehl V, Schultz-Coulon HJ, Zur Hausen $\mathrm{H}$. Molecular cloning and characterization of human papilloma virus DNA derived from a laryngeal papilloma. J Virol 1982; 44: 393-400.

13. Dartmann K, Schwarz E, Gissmann L, Zur Hausen H. The nucleotide sequence and genome organization of human papilloma virus Type 11. Virology 1986; 151: 124-30. 